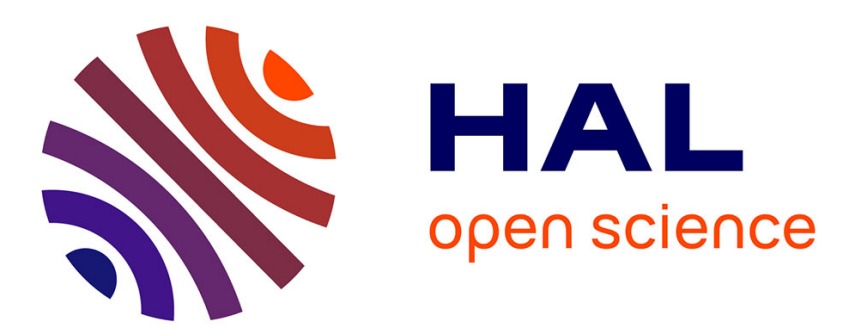

\title{
Perturbation singulière d'un problème de frottement sec non monotone
}

\author{
Yves Renard
}

\section{To cite this version:}

Yves Renard. Perturbation singulière d'un problème de frottement sec non monotone. Comptes rendus de l'Académie des sciences. Série I, Mathématique, 1998, 326, pp.131-136. 10.1016/S07644442(97)82726-7 . hal-00690534

\section{HAL Id: hal-00690534 https://hal.science/hal-00690534}

Submitted on 25 Apr 2018

HAL is a multi-disciplinary open access archive for the deposit and dissemination of scientific research documents, whether they are published or not. The documents may come from teaching and research institutions in France or abroad, or from public or private research centers.
L'archive ouverte pluridisciplinaire HAL, est destinée au dépôt et à la diffusion de documents scientifiques de niveau recherche, publiés ou non, émanant des établissements d'enseignement et de recherche français ou étrangers, des laboratoires publics ou privés. 


\section{Perturbation singulière d'un problème de frottement sec non monotone}

\section{Yves RENARD}

Laboratoire de Modélisation el Calcul, URA-CNRS 397, B.P. 53X, 38041 Grenoble cedex, France E-mail: Yves.Renard@imag.fr

Résumé. Dans cette Note on étudie un problème de perturbation singulière constitué d'une inclusion différentielle qui admet une solution unique pour toute valeur du paramètre de perturbation. Le problème dégénéré, qui correspond à un problème de frottement sec dynamique, admet une multitude de solutions. On montre que la solution du problème perturbé converge simplement vers une solution particulière du problème dégénéré lorsque le paramètre de perturbation tend vers zéro. L'approche par perturbation singulière permet une analyse d'un critère de choix utilisé pour la sélection d'une solution du problème dégénéré, et suggère une méthode pour aborder des problèmes de frottement plus élaborés.

\section{Singular perturbation of a non-monotonous dry friction problem}

Abstract. In this Note we deal with a singularly perturbed system constituted by a differential inclusion which has a unique solution for each value of the perturhation parameter. The associated degenerated problem, that corresponds to a dynamic dry friction problem, has many solutions. We show that perturbed problem solutions converge to a particular solution of the degenerated problem when the perturbation parameter goes to zero. The singular perturbation approach allows an analysis of a criterion used to select a solution of the degenerated problem, and suggests a method to study more claborated dry friction problems.

\section{Abridged English Version}

We consider the perturbation problem of finding $v_{\varepsilon}:[0, T] \rightarrow \mathbb{R}$, absolutely continuous solution of (1), with $\varepsilon>0$ the perturbation parameter, and $\beta: \mathbb{R} \longrightarrow \mathcal{P}(\mathbb{R})$ a multi-valued map satisfying the two following assumptions:

HYPOTHESIS 1. $-\beta$ is upper semi-continuous with compact convex values and $(-\beta)$ is semi-Lipschitz

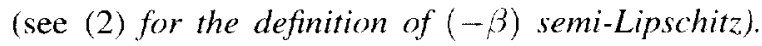


Hypothesis 2. - There exist $N \geq 0$ and $2 N$ pairs $\left(v_{j}^{*}, \alpha_{j}^{*}\right)_{1 \leq j \leq 2 N}$, with $v_{1}^{*} \leq v_{2}^{*} \leq \cdots \leq v_{2 N}^{*}$ and:

- $\left.\left.\left.]-\infty, v_{1}^{*}\right],\right]-\infty, \alpha_{1}^{*}\right] ;\left[v_{2 i}^{*}, v_{2 i+1}^{*}\right],\left[\alpha_{2 i}^{*}, \alpha_{2 i+1}^{*}\right], 1 \leq i<N ;$ and $\left[v_{2 N}^{*},+\infty\left[,\left[\alpha_{2 N}^{*},+\infty[\right.\right.\right.$ strictly increasing branches of $\beta$,

- $\left[v_{2 i-1}^{*}, v_{2 i}^{*}\right],\left[\alpha_{2 i-1}^{*}, \alpha_{2 i}^{*}\right], 1 \leq i \leq N$, strictly decreasing branches of $\beta$, where $\left[v_{1}, v_{2}\right],\left[\alpha_{1}, \alpha_{2}\right]$ is called a strictly increasing (resp. strictly decreasing) branch of $\beta$ if $\alpha_{1} \in \beta\left(v_{1}\right), \alpha_{2} \in \beta\left(v_{2}\right), \alpha_{1}<\alpha_{2}$ (resp. $\left.\alpha_{1}>\alpha_{2}\right)$ and:

$$
(\phi-\psi)(u-w)>0 \text { (resp. }<0) \forall u, w \in] v_{1}, v_{2}[, u \neq w \forall \phi \in \beta(u), \forall \psi \in \beta(v)
$$

Hypothesis 1 is sufficient for the problem (1) to have a unique a.c. solution (which is moreover Lipschitz, see [3]), and hypothesis 2 is sufficient to establish the convergence results presented in this Note.

Terminology. - A point $(\alpha, v)$ with $\alpha \in \beta(v)$ is called stable point (resp. unstable point) if there exists a strictly increasing (resp. decreasing) branch $\left[v_{1}, v_{2}\right],\left[\alpha_{1}, \alpha_{2}\right]$ of $\beta$ with $\left.\alpha \in\right] \alpha_{1}, \alpha_{2}[$ (resp. $\alpha \in]\left(\alpha_{2}, \alpha_{1}[)\right.$ and $v \in\left[v_{1}, v_{2}\right]$. The points $\left(\alpha_{2 i-1}^{*}, v_{2 i-1}^{*}\right)$ are called maximum critical points; the points $\left(\alpha_{2 i}^{*}, v_{2 i}^{*}\right)$ and minimum critical points.

Problem (3) is called the degenerated problem. Generally, when $\beta$ is non-monotonous, there are many solutions of this problem. In Ionescu and Paumier [5] and Leonov and Srinivasan [6] one can see how the friction of an horizontal homogeneous elastic slab can be modelized with problem (3), where $v_{0}$ is the slip velocity on the contact boundary. The use of a non-monotonous slip dependent friction coefficient leads to a non-monotonous map $\beta$. The first result is that sequence $v_{\varepsilon}$ is bounded:

Proposition 1. - For all $\varepsilon>0, \alpha \in \mathrm{BV}(0, T)$, and $\beta$ satisfying hypothesis 1 and 2 , the unique solution $v_{\varepsilon}$ of the problem (1) satisfies:

$$
v_{\varepsilon} \in \mathrm{L}^{\infty}(0, T) \text { with }\left\|v_{\varepsilon}\right\|_{\mathrm{L}^{\infty}(0, T)}<M_{1} \text {, where } M_{1}>0 \text { is a constant which is independent of } \varepsilon
$$

We introduce the following definition:

Definition. $\quad \Lambda$ point $v \in \mathbb{R}$ is an attractive point of the boundary layer at $t=0$ if $\alpha^{+}(0) \in \beta(v)$ and if there exist a strictly increasing branch $\left[v_{1}, v_{2}\right],\left[\alpha_{1}, \alpha_{2}\right]$ of $\beta$ and $\zeta>0$ such that:

$$
v \in\left[v_{1}, v_{2}\right], \quad \alpha^{+}(t) \in\left[\alpha_{1}, \alpha_{2}\right] \quad \forall t \in[0, \zeta]
$$

The analysis of the boundary layer of the problem is essential in the proof of the following main result:

THгоRem 1. - With $\beta$ a multi-valucd map satisfying hypothesis 1 and $2, \alpha \in \mathrm{BV}(0, T)$, and $\left(\alpha^{+}(0), v^{0}\right)$ not an unstable point, the solutions sequence $v_{s}$ of Problem (1) pointwise converges to a function $v_{u}$ when $\varepsilon$ goes to zero. Moreover, $v_{0}$ is continuous to the left, has a limit to the right everywhere on $] 0, T]$, and satisfies:

$$
\left.\left.\alpha^{-}(t) \in \beta\left(v_{0}(t)\right) \forall t \in\right] 0, T\right], \quad v_{0}^{+}(t) \text { is an attractive point for all } t \in[0, T[
$$

If we consider the criterion proposed by Ionescu and Paumier [5] to select a particular solution of Problem (3), which is:

the system only jumps when it has no other choice,

it can be shown that if this criterion selects a unique solution of Problem (3) this is the same as the limit solution of Theorem 1.

One can find a detailed proof of these results in [9]. 


\section{Introduction}

Le problème du glissement dynamique d'une couche élastique homogène avec frottement de Coulomb et coefficient de frottement dépendant de la vitesse de glissement, est utilisé pour la modélisation des mouvements «stick-slip », par exemple pour l'étude du glissement de failles géologiques (voir [1] et [2]). Ce genre de dépendance, introduite en particulier par E. Rabinowicz [8] (voir aussi [4]) fait apparâtre une multiplicité des solutions quand elle est appliquée à un solide élastique. Cela rend nécessaire un critère de choix de la solution physiquement acceptable (voir [5] et [6]). L'approche par perturbation singulière adoptée ici permet une analyse d'un critère de choix, et donne une méthode pour aborder des problèmes de frottement plus complexes. On trouvera une démonstration détaillée des résultats annoncés dans cette Note dans [9].

\section{Définition du problème}

Soit le problème de perturbation singulière consistant à trouver $v_{\varepsilon}:[0, T] \longrightarrow \mathbb{R}$ absolument continue vérifiant :

$$
\left\{\begin{array}{l}
\varepsilon \frac{\mathrm{d}}{\mathrm{d} t} v_{\varepsilon}(t) \in \alpha(t)-\beta\left(v_{\varepsilon}(t)\right) \quad \text { pour presque tout } t \in[0, T] \\
v_{\varepsilon}(0)=v^{0}
\end{array}\right.
$$

où $\varepsilon>0$ est le paramètre de perturbation, $\alpha(t):[0, T] \rightarrow \mathbb{R}$ est à variation bornée (on écrira $\alpha \in \mathrm{BV}(0, T)$ ) et $\beta: \mathbb{R} \longrightarrow \mathcal{P}(\mathbb{R})$ est une application multivoque sur laquelle on impose deux types de conditions indiquées dans les hypothèses 1 et 2 qui suivent.

HyPOTHÈse 1. - $\beta$ est semi-continue supérieurement à valeurs compactes convexes et $(-\beta)$ est semi-lipschitzienne, c'est-à-dire qu'il existe une constante $M_{\beta}$ telle que :

$$
(\phi-\psi)\left(v_{1}-v_{2}\right) \leq M_{i 3}\left(v_{1}-v_{2}\right)^{2} \quad \forall v_{1}, v_{2} \in \mathbb{R}, \forall \phi \in \beta\left(v_{2}\right), \forall \psi \in \beta\left(v_{1}\right)
$$

On dit qu'une branche $\left[v_{1}, v_{2}\right],\left[\alpha_{1}, \alpha_{2}\right]$ est strictement croissante (resp. strictement décroissante) si $\alpha_{1} \in \beta\left(v_{1}\right), \alpha_{2} \in \beta\left(v_{2}\right), \alpha_{1}<\alpha_{2}$ (resp. $\left.\alpha_{1}>\alpha_{2}\right)$ et :

$$
(\phi-\psi)(u-w)>0(\operatorname{resp}<0) \forall u, w \in] v_{1}, v_{2}[, u \neq w \quad \forall \phi \in \beta(u), \forall \psi \in \beta(w)
$$

HYPOTHĖsE 2 . - Il existe $N \geq 0$ et $2 N$ couples $\left(v_{j}^{*}, \alpha_{j}^{*}\right)_{1 \leq j \leq 2 N}$, avec $v_{1}^{*} \leq v_{2}^{*} \leq \cdots \leq v_{2 N}^{*}$ et :

- ] $\left.\left.\left.-\infty, v_{1}^{*}\right],\right]-\infty, \alpha_{1}^{*}\right] ;\left[v_{2 i}^{*}, v_{2 i+1}^{*}\right],\left[\alpha_{2 i}^{*}, \alpha_{2 i+1}^{*}\right], 1 \leq i<N ;$ et $\left[v_{2 N}^{*},+\infty\left[,\left[\alpha_{2 N}^{*},+\infty[\right.\right.\right.$ branches strictement croissantes de $\beta$,

- $\left[v_{2 i-1}^{*}, v_{2 i}^{*}\right],\left[\alpha_{2 i-1}^{*}, \alpha_{2 i}^{*}\right], 1 \leq i \leq N$, branches strictement décroissantes de $\beta$.

La condition de semi-continuité supérieure est suffisante à l'existence de solutions absolument continues au problème (1). Une application multivoque $\beta$ étant semi-continue supéricurement si pour tout fermé $A \subset \mathbb{R}$ l'ensemble $\beta^{-1}(A) \subset \mathbb{R}$ est fermé. La condition $\beta$ semi-lipschitzienne assure l'unicité de la solution et, avec $\alpha$ bornée, donne une régularité lipschitzienne pour la solution (voir [3]). L'hypothèse 2 est suffisante pour établir les résultats de convergence qui suivent.

Terminologie. - Un point $(\alpha, v)$ avec $\alpha \in \beta(v)$ est appelé point stable (resp. point instable) s'il existe une branche strictement croissante (resp. décroissante) $\left[v_{1}, v_{2}\right],\left[\alpha_{1}, \alpha_{2}\right]$ avec $\left.\alpha \in\right] \alpha_{1}, \alpha_{2}[$ (resp. $\alpha \in] \alpha_{2}, \alpha_{1}[)$ et $v \in\left[v_{1}, v_{2}\right]$. Les points $\left(\alpha_{j}^{*}, v_{j}^{*}\right)$ sont appelés les points critiques dont les points $\left(\alpha_{2 i-1}^{*}, v_{2 i-1}^{*}\right)$ sont les points critiques maximums et les $\left(\alpha_{2 i}^{*}, v_{2 i}^{*}\right)$ les points critiques minimums. 
On appelle problème dégénéré le problème suivant :

$$
\left\{\begin{array}{l}
\text { Trouver } \left.\left.v_{0}:\right] 0, T\right] \longrightarrow \mathbb{R} \text { telle que : } \\
\left.\left.\left.\alpha^{-}(t) \in \beta\left(v_{0}(t)\right) \forall t \in\right] 0, T\right] \quad{ }^{1}\right)
\end{array}\right.
$$

Ce problème, qui est scalaire, n'admet une solution unique, pour une donnée $\alpha$ quelconque dans $\mathrm{BV}(0, T)$, que si l'application $\beta$ est strictement monotone.

\section{Relation avec le problème de frottement}

On considère une couche élastique fixée au plan rigide immobile $y=H$, et glissant avec frottement sur le plan rigide $y=0$ qui est en mouvement suivant l'axe des $x$ avec une vitesse $V_{c}$ (voir figure la). $\mathrm{Si}$ on se limite aux solutions indépendantes des coordonnées horizontales, le déplacement $u$ suivant l'axe des $x$ est gouverné par les équations suivantes:

$$
\begin{array}{r}
\left.\left.\partial_{t t}^{2} u(t, y)-c^{2} \partial_{y y}^{2} u(t, y)=0, \quad y \in\right] 0, H[, \quad l \in] 0, T\right] \\
\left.\left.u(t, H)=0, \quad G \partial_{y} u(t, 0)=-F_{f}, \quad t \in\right] 0, T\right]
\end{array}
$$

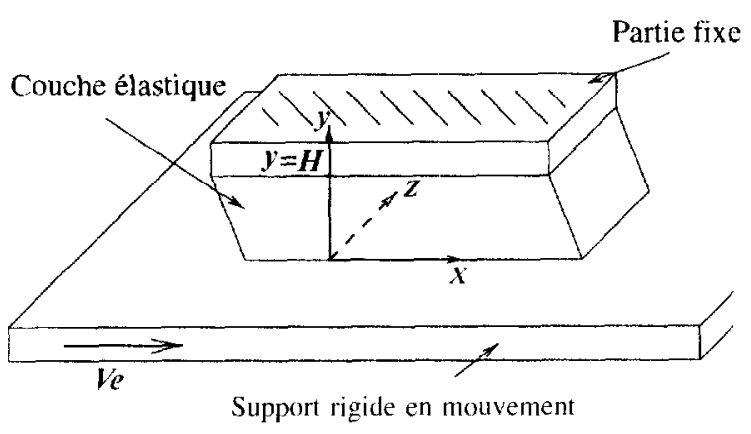

Figure la. - Couche élastique entrainée.

Figure la. - Elastic slab on a moving foundation

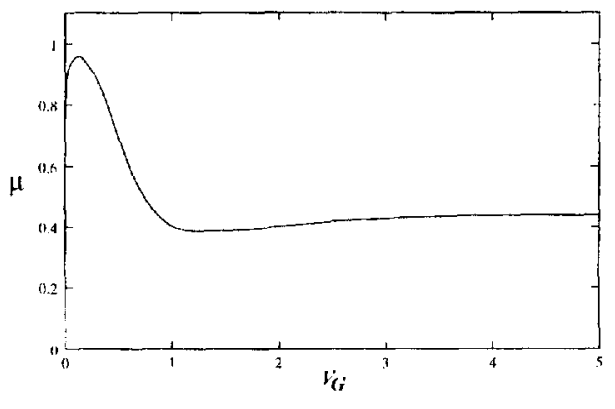

Figure Ib. - Exemple de coefficient de frottement.

Figure th. - Exomple of friction coefficient.

où $F_{f}$ est la force de frottement, $G$ le module de cisaillement élastique, $c=\sqrt{G / \rho}$ la vitcsse des ondes et $\rho$ la densité. Le contact entre la couche élastique et le plan rigide $y=0$ est modélisé par une loi de Coulomb avec coefficient de frottement dépendant de la vitesse de glissement, qui s'écrit :

$$
F_{f} \in-S \mu\left(\left|v_{(i}\right|\right) \operatorname{Sgn}\left(v_{c i}\right)
$$

où $\mu: \mathbb{R}^{+} \longrightarrow \mathbb{R}^{+}$est le coefficient de frottement qui dépend de la vitesse de glissement (la figure $1 \mathrm{~b}$ donne un exemple typique de dépendance de ce coefficient), $S$ la pression de contact que l'on suppose constante, $v_{c i}=\partial_{t} u(t, 0)-V_{r}(t)$ la vitesse de glissement sur le bord de contact et $\operatorname{Sgn}(v)$ la fonction signe multivoque qui vaut tout l'intervalle $[-1,1]$ en $v=0$ et $\{v /|v|\}$ ailleurs. On complète le problème par les conditions initiales :

$$
u(0, y)=u_{0}(y) . \quad \partial_{t} u(0, y)=u_{1}(y), \quad y \in[0, H]
$$

(') On note les limites à droite et à gauche respectivement $v^{+}(t)=\lim _{\tau \rightarrow t^{+}} v(\tau)$ et $v^{-}(t)=\lim _{\tau \rightarrow t^{-}} v(\tau)$ 
En suivant Ionescu et Paumier [5], ou Leonov et Srinivasan [6], on ramène le problème (4)-(7) sur le bord de contact, grâce à des égalités sur les droites caractéristiques. Ainsi, en posant :

$$
\alpha(t)=\frac{G}{c}\left(u_{1}(c t) \quad V_{e}(t)\right) \mid G \partial_{y} u_{0}(c t), \quad \beta(v)=\frac{G}{c} v+S \mu(|v|) \operatorname{Sgn}(v)
$$

le problème revient, pour $T<\frac{H}{c}$, à trouver $v_{G i}(t)$ telle que :

$$
\alpha(t) \in \beta\left(v_{G}(t)\right)
$$

c'est-à-dire à inverser l'inclusion (9), ce qui entre bien dans le cadre du problème (3). En général, ce problème admet une infinité de solutions; c'est pourquoi Ionescu et Paumier [5] proposent un critère de choix de solution issu de la théorie des catastrophes, dit critère du retard maximal, qui s'énonce ainsi :

Le système effectue un saut en vitesse quand il n'a plus d'autres choix possibles

Indépendamment, Leonov et Srinivasan [6] proposent un critère similaire.

\section{Principaux résultats}

Majoration. - Par un raisonnement élémentaire, on montre que s'il existe $\left[v_{1}, v_{2}\right],\left[\alpha_{1}, \alpha_{2}\right]$ tels que $\alpha_{1} \in \beta\left(v_{1}\right), \alpha_{2} \in \beta\left(v_{2}\right), v^{0} \in\left[v_{1}, v_{2}\right]$ et $\alpha(t) \in\left[\alpha_{1}, \alpha_{2}\right]$ sur $[0, T]$, alors la solution $v_{\varepsilon}$ du problème (1) vérifie $v_{\varepsilon}(t) \in\left[v_{1}, v_{2}\right]$ sur $[0, T]$ pour tout $\varepsilon>0$. Ceci nous permet d'établir le résultat suivant :

Proposition. - Pour tout $\varepsilon>0, \alpha \in \mathrm{BV}(0, T)$ et $\beta$ vérifiant les hypothèses 1 et 2, I'unique solution $v_{\varepsilon}$ du problème (1) vérifie :

$$
v_{\varepsilon} \in \mathrm{L}^{\infty}(0, T) \text { avec }\left\|v_{\varepsilon}\right\|_{\mathrm{L}^{\infty}(0, T)}<M_{1} \text {, où } M_{1}>0 \text { est une constante indépendante de } \varepsilon
$$

Comportement au voisinage de $t=0$. - Dans le cas le plus courant où la condition initiale ne vérifie pas $\alpha^{+}(0) \in \beta\left(v^{0}\right)$, on a un comportement de couche limite en $t=0$ lorsque $\varepsilon$ tend vers zéro. Nous nous sommes inspiré d'une étude de A.N. Tikhonov, A.B. Vasiléva et A.G. Sveshnikov [10] qui concerne un cas plus régulier. Nous introduisons les notions suivantes :

DÉfinitions. - Soit $v_{\varepsilon}$ la suite des solutions du problème (1) pour $\varepsilon>0$.

- On dit que $v \in \mathbb{B}$ est un point d'adhérence de la couche linite en $t=0$ si et seulement s'il existe une suite $\left(\varepsilon_{n}\right)_{n \geq 0}$ strictement positive et une suite $\left(t_{n}\right)_{n \geq 0}$ positivę telles que $: \lim _{n} \rightarrow+\infty \varepsilon_{n}=0$, $\lim _{n} \rightarrow+\infty t_{n}=0, \lim _{n} \rightarrow+\infty v_{\varepsilon_{n}}\left(l_{n}\right)=v$. L'ensemble des points d'adhérence qui correspondent à une donnée initiale $v^{0}$ est un intervalle fermé borné que l'on note $\mathrm{I}_{a}\left(v^{0}\right)$.

- On dit que $v \in \mathbb{R}$ est un point limite de la couche limite en $t=0$ si et seulement s'il existe $t_{\varepsilon}>0$ pour tout $\varepsilon>0$ tel que $: \lim _{\varepsilon \rightarrow 0} t_{\varepsilon}=0, \lim _{\varepsilon \rightarrow 0} v_{\varepsilon}\left(t_{\varepsilon}\right)=v$. L'ensemble des points limites qui correspondent à une donnée initiale $v^{0}$ est un sous-intervalle fermé de $\mathrm{I}_{a}\left(v^{0}\right)$ que l'on note $\mathrm{I}_{l}\left(v^{0}\right)$.

- On dit que $v \in \mathbb{R}$ est un point attractif de la couche limite en $t=0$ si $\alpha^{+}(0) \in \beta(v)$ et si de plus il existe une branche strictement croissante $\left[v_{1}, v_{2}\right]:\left[\alpha_{1}, \alpha_{2}\right]$ de $\beta$ et $\zeta>0$ telle que :

$$
v \in\left[v_{1}, v_{2}\right], \quad \alpha^{+}(t) \in\left[\alpha_{1}, \alpha_{2}\right] \quad \forall t \in[0, \zeta]
$$

Par une suite de résultats élémentaires on montre que si $\left(\alpha^{+}(0), v^{0}\right)$ n'est pas un point instable de $\beta$, alors on peut décrire complètement les intervalles $\mathbf{I}_{a}\left(v^{0}\right)$ et $\mathrm{I}_{l}\left(v^{0}\right)$. 
On montre ainsi que lorsque $\left(\alpha^{+}(0), v^{0}\right)$ n'est pas un point instable, l'intervalle $\mathbf{I}_{l}\left(v^{0}\right)$ contient un et un seul point attractif. On peut alors caractériser le comportement de la suite $v_{\varepsilon}$ au voisinage de $t=0$ de la manière suivante :

Proposition 2. - Avec $\alpha \in \mathrm{BV}(0, T), \beta$ qui satisfait les hypothèses 1 et 2 et le point $\left(\alpha^{+}(0), v^{0}\right)$ qui n'est pas un point instable de $\beta$, il existe une branche strictement croissante $\left[v_{1}, v_{2}\right],\left[\alpha_{1}, \alpha_{2}\right]$ de $\beta$ et $\zeta>0$ tels que la suite $v_{\varepsilon}$ des solutions du problème (1) converge simplement vers une fonction $v_{0}$ sur $[0, \zeta]$ quand $\varepsilon$ tend vers zéro. De plus, $v_{0}$ est continu à gauche, a une limite à droite partout sur $] 0, \zeta]$ et vérifie pour tout $t \in] 0, \zeta[$ :

$$
\alpha^{+}(t) \in\left[\alpha_{1}, \alpha_{2}\right], \quad \alpha^{-}(t) \in \beta\left(v_{0}(t)\right), \quad v_{0}^{+}(t) \in\left[v_{1}, v_{2}\right], \quad v_{0}^{+}(t) \text { est un point attractif }
$$

Résultat de convergence. - Il est alors possible de montrer le résultat suivant :

THÉORÈmE. - Pour une application $\beta$ vérifiant les hypothèses 1 et $2, \alpha \in \mathrm{BV}(0, T)$ et $\left(\alpha^{+}(0), v^{0}\right)$ $n$ 'étant pas un point instable de $\beta$, la suite des solutions $v_{\varepsilon}$ du problème (1) converge simplement vers une fonction $v_{0}$ quand $\varepsilon$ tend vers zéro. De plus, $v_{0}$ est continu à gauche, a une limite à droite partout sur $] 0, T]$ et vérifie :

$$
\left.\left.\alpha^{-}(t) \in \beta\left(v_{0}(t)\right) \forall t \in\right] 0, T\right], \quad v_{0}^{+}(t) \text { est un point attractif pour tout } t \in[0, T[
$$

La démonstration de ce résultat passe par l'application successive de la proposition 2 sur un nombre fini d'intervalles. La deuxième propriété énoncée implique que la solution limite reste sur les branches strictement croissante de $\beta$.

Relation avec le critère de retard maximal. - L'hypothèse 2 n'est pas trop restrictive pour traiter des problèmes de frottement. En effet, les dépendances choisies dans les modélisations (voir [4] et [7]) sont toujours très régulières. Si on considère le critère du retard maximal défini par $(10)$, on peut montrer que lorsque celui-ci permet de sélectionner une solution unique au problème (3), alors celle-ci cöncide avec la solution limite du théorème 1. Malgré tout, il n'y a pas une totale équivalence car l'énoncé (10) n'est pas suffisamment précis pour sélectionner une solution unique dans tous les cas de figure. Cette étude apporte un éclairage sur l'utilisation de ce critère. Elle donne aussi des perspectives pour des problèmes de frottement bidimensionnels ou tridimensionnels où l'approche par perturbation singulière est envisageable alors qu'il semble plus difficile d'exhiber des critères de choix.

\section{Références bibliographiques}

[1] W.R. Brace, J.D. Byerlee. Stick-slip as a mechanism for earth-quakes, Science, 153 (1966), 990-992.

[2] M. Campillo, I.R. Ionescu, J.-C. Paumier, Y. Renard. On the dynamic sliding with friction of a rigid block and of an infinite elastic slab. Physics of the Earth and Planetary Interiors, 96 (1996), 15-23.

[3] K. Deimling. Multivalued differential equations, Walter de Gruyter, Berlin, 1992.

[4] C. Gao, D. Kuhlmann-Wilsdorf. On stick-slip and velocity dependence of friction at low speeds, ASME J. Tribology, $11(1990), 355-360$.

[5] I.R. Ionescu, J.-C. Paumier. On the contact problem with slip rate dependent friction in elastodynamic, Eur. J. Mech., A/Solids, 4 (1994), 555-568.

[6] A.I. Leonov, A. Srinivasan. Self-Oscillations of an Elastic Plate Sliding over a Smooth Surface, Int. J. Engng. Sci., 31 (1993), 453-473.

[7] Li Chun Bo, D. Pavelescu. The friction-speed relation and its influence on the critical velocity of stick-slip motion, Wear, 82 (1982), 277-289.

[8] F. Rabinowicz. The intrinsic variables affecting the stick-slip process, Proc. Roy. Phys. Soc., 71 (1958), 668-675.

[9] Y. Renard, Singular perturbation approach of an elastic dry friction problem with a non-monotonous friction coefficient, submitted.

[10] A.N. Tikhonov, A.B. Vasiléva, A.G. Sveshnikov. Differential Equations, Chapter VII, Asymptotics of solutions of differential equations with respect to a small parameter, 181-213, Springer Verlag, 1980. 